\title{
Rheumatic disorders in Zimbabwe: a prospective analysis of patients attending a rheumatic diseases clinic
}

Michael Stein, Paul Davis

\begin{abstract}
The pattern of rheumatic disease in Africa differs from that in Europe and the United States and these differences may provide clues to its cause or pathogenesis. In a six month prospective analysis of 141 patients (83 female) attending a rheumatic diseases clinic rheumatoid arthritis was the commonest disorder, occurring in 49 patients. Twenty seven of the $49(55 \%)$ were seropositive, $25(51 \%)$ had erosive disease with rheumatoid nodules $(13 / 49,27 \%)$, and extra-articular complications $(6 / 49,12 \%)$, indicating a pattern of disease unlike the early reports from Africa. Systemic lupus erythematosus found in 18/141 (13\%), gout in $12(9 \%)$, ankylosing spondylitis in six (4\%), and Reiter's syndrome in five $(4 \%)$, in contrast with their rarity in previous reports from Africa, were not uncommon, whereas tropical polyarthritis was seldom diagnosed. The pattern of rheumatic disease in Harare, a large city, is changing to approximate more closely the pattern seen in developed countries.
\end{abstract}

The pattern of rheumatic diseases in Africa has aroused interest as differences in prevalence and clinical features may provide clues to causation and pathogenesis. ' Rheumatoid arthritis (RA) was thought to be uncommon in Africa, with Hall reporting seven cases in 10000 hospital admissions from Kenya in $1962,{ }^{2}$ and the illness apparently not described in black South Africans before $1970 .^{3}$ Greenwood subsequently noted that in Nigerians RA was a milder illness with a rarity of nodules, vasculitis, and radiological erosions. ${ }^{4}$ More recent reports from Africa, however, have found the prevalence of RA in an urban population to be similar to that in Western Europe, ${ }^{5}$ with severe disease more prominent than in earlier reports. ${ }^{6-8}$ Similarly, systemic lupus erythematosus (SLE) was excessively rare in Africa, with the illness unrecorded before $1960^{\circ}$ and single case reports of small series of patients subsequently reported, but it is now reported more often. ${ }^{10}$

There are two studies of the pattern of rheumatic diseases in Zimbabwe. Gelfand in 1969 described patients admitted to medical wards with arthritis ${ }^{11}$ and Lutalo in 1985 reported the pattern of chronic inflammatory rheumatic diseases in patients on a chronic diseases register. ${ }^{12}$ Our experience differs from these descriptions and most other reports from Africa.

Dr Michael Stein

Department of Clinical

Pharmacology, University

of Zimbabwe, PO Box A 178

of Zimbabwe, PO Box A 178,
Avondale, Harare, Zimbabwe.

Accepted for publication

21 July 1989

Patients and methods

We prospectively studied all patients attending a rheumatic diseases clinic for six months at Parirenyatwa Hospital, a major referral hospital in Harare. Health services in Zimbabwe extend to the most remote parts of the country with most patients initially seen at primary health care clinics by specially trained registered nurses. Problems are referred either to the clinic doctor or to a hospital. Specialist services are concentrated in the large central hospitals of Bulawayo and Harare and patients are referred from distant hospitals, from primary health care clinics within the cities, from general practitioners, and from outpatient or casualty services within the hospitals. At the time of the study this was the only specialist rheumatic diseases clinic, but many patients with rheumatic diseases are managed by other health workers throughout the country. Patients from both urban and rural backgrounds were referred to the clinic by colleagues aware of our interest in inflammatory rheumatic disease.

Patients were assessed by one of the authors, appropriate serological investigations performed, and $x$ rays obtained of relevant joints. Patients with RA fulfilled the criteria for diagnosis, ${ }^{13}$ patients with SLE fulfilled the American Rheumatism Association preliminary criteria,${ }^{14}$ patients with Reiter's syndrome fulfilled the criteria proposed by Calin, ${ }^{15}$ and patients with ankylosing spondylitis fulfilled the New York criteria. ${ }^{16}$ As electromyography and muscle biopsies are seldom performed in our patients with dermatomyositis and polymyositis, criteria for diagnosis were classical clinical findings and markedly raised muscle enzymes. The diagnosis of gout was made in patients with uric acid crystals in the synovial fluid or those with a classical history and radiological changes typical of gout. Functional class was assessed by a local modification of the American Rheumatism Association gradings ${ }^{17}$ : class I-normal function; II-normal function, though with pain and difficulty; IIIa-ability to do paid work or housework diminished but self sufficient in activities relating to personal hygiene and care; IIIb-as for IIIa but not self sufficient; and IV-chair or bedridden.

\section{Results}

Table 1 shows the diagnosis and sex distribution of the patients seen and table 2 the clinical features of the 49 patients with RA. The average duration of disease in patients with RA was 5.5 (SD 4.9) years and the pattern of joint involvement was small joints of the hand in 43/49 $(88 \%)$, wrist in $38(78 \%)$, knee in $30(61 \%)$, 
Table 1: Age and sex distribution of patients attending a rheumatic diseases clinic in Zimbabwe

\begin{tabular}{lrrrlr}
\hline Diagnosis & $\begin{array}{l}\text { Total } \\
\text { number }\end{array}$ & Men & Women & $\begin{array}{l}\text { Onset age } \\
\text { in years (SD) }\end{array}$ & \% Of total \\
\hline Rheumatoid arthritis & 49 & 18 & 31 & $38 \cdot 9(11 \cdot 9)$ & 35 \\
Osteoarthritis & 5 & 0 & 5 & $58 \cdot 0(11 \cdot 7)$ & 4 \\
Gout & 12 & 11 & 1 & $51 \cdot 6(8 \cdot 6)$ & 9 \\
Juvenile chronic arthritis & 6 & 1 & 5 & $9 \cdot 8(4 \cdot 9)$ & 4 \\
Systemic lupus erythematosus & 18 & 0 & 18 & $30 \cdot 8(11 \cdot 7)$ & 13 \\
Dermatomyositis & 5 & 0 & 5 & $23 \cdot 4(12 \cdot 8)$ & 4 \\
Scleroderma & 3 & 1 & 2 & $34 \cdot 0(9 \cdot 2)$ & 2 \\
Reiter's syndrome & 5 & 4 & 1 & $28 \cdot 2(2 \cdot 1)$ & 4 \\
HIV reactive arthritis & 12 & 11 & 1 & $32 \cdot 8(6 \cdot 0)$ & 9 \\
Ankylosing spondylitis & 6 & 5 & 1 & $35 \cdot 8(12 \cdot 7)$ & 4 \\
Unclassified acute & 4 & 1 & 3 & $35 \cdot 7(15 \cdot 2)$ & 3 \\
Unclassified chronic & 5 & 1 & 4 & NA & 4 \\
Miscellaneous & 11 & 5 & 6 & NA & 8 \\
Total & 141 & 58 & 83 & & \\
\hline
\end{tabular}

Table 2: Clinical features in 49 patients with rheumatoid arthritis

\begin{tabular}{ll}
\hline Clinical feature & $\begin{array}{l}\text { Prevalence } \\
\text { No }(\%)\end{array}$ \\
\hline Seropositive & $27(55)$ \\
Radiological erosions & $25(51)$ \\
Deformities & $15(31)$ \\
Nodules & $13(27)$ \\
Lung involvement & $4(8)$ \\
Vasculitis & $3(6)$ \\
Sjögren's syndrome & $1(2)$ \\
Neuropathy & $1(2)$ \\
Functional class: & $12(25)$ \\
I & $24(49)$ \\
II & $7(14)$ \\
IIIa & $5(10)$ \\
IIIb & $1(2)$ \\
IV & \\
\hline
\end{tabular}

in $15(31 \%)$, feet in $10(20 \%)$, cervical spine in eight $(16 \%)$, and hip in four $(8 \%)$. Antinuclear antibodies were present in $15 / 49(31 \%)$ patients with RA. Juvenile chronic arthritis with systemic (two patients), polyarticular seropositive (two patients), polyarticular seronegative (one patient), and pauciarticular onset (one patient) was seen. Antinuclear antibodies were not detected, nor did eye involvement occur. Three patients (class IIIb two patients, class IV one patient) were severely handicapped.

Osteoarthritis was seen in five female patients. The knee was the most commonly affected joint (4/5). No patient had Heberden's nodes or involvement of the hip joint. Gout (12 patients) occurred predominantly in middle aged men. Uric acid crystals were seen in the synovial fluid of seven patients $(58 \%)$ and tophi in two $(17 \%)$. Other clinical features were a history of heavy alcohol use in $6 / 12(50 \%)$, obesity in seven $(58 \%)$, diuretic use in three $(25 \%)$, and hypertension in five $(42 \%)$. Joints most commonly involved were the first metatarsophalangeal joint in $10 / 12(83 \%)$, knee in $10(83 \%)$, and ankle in seven $(58 \%)$.

We have previously given details of the clinical features of patients with SLE in Zimbabwe, ${ }^{10}$ and the findings in this study were similar. Dermatomyositis was not associated with malignancy in any patient and the clinical features were similar to the classical descriptions. Clinical features of the spondyloarthropathies ${ }^{18}$ and a reactive arthritis associated with the human immunodeficiency virus ${ }^{19}$ have been described elsewhere.
The five patients with unclassified chronic arthritis did not fulfil the diagnostic criteria used. Probable gout (one patient) and probable rheumatoid arthritis (four) were the diagnoses. Conditions under the heading 'miscellaneous' (one patient each) were rheumatic fever, metabolic bone disease, fibromyalgia, haemophilia, bone cyst, palindromic rheumatism, frozen shoulder, erythema nodosum, pseudogout, tuberculosis, and a Charcot's joint.

\section{Discussion}

Although this study was performed prospectively over six months, many of the patients had been attending the hospital before the study period and as it presents the pattern of rheumatic illness seen at a specialist, hospital based clinic it would be inappropriate to infer prevalence or incidence data. Rheumatoid arthritis, as in other studies, 241112 was the commonest illness seen, and in keeping with the more recent reports $^{6-8}$ followed the pattern of illness seen in Europe more closely, with seropositive, destructive arthritis, and a female preponderance, rather than the milder pattern described by Greenwood. ${ }^{4}$ The prevalence of RA as well as the severity of illness is increased in urban African populations compared with their rural counterparts. ${ }^{5}$ One explanation for the differences between the original reports and the more recent ones is a changing pattern of disease due to increased urbanisation.

There are no formal epidemiological studies of the prevalence of SLE in urban and rural African populations. The pattern of recognition has been similar to that for RA, with the illness unrecognised initially and then reported more and more often. Systemic lupus erythematosus in Zimbabwe is no longer a rare disease and we have recently reported the largest series from Africa. ${ }^{10}$ These changes in the pattern of disease are unlikely to be due to altered diagnostic skills as the early formal medical services in most African countries were provided by expatriate doctors with a training emphasis in these illnesses. Gelfand did not find a single case of SLE in 1969 in a central hospital in Harare. ${ }^{11}$ More recently (1985), Lutalo in a district hospital in Gweru, Zimbabwe found no patients with SLE on a chronic diseases register. ${ }^{12}$ This adds weight to the argument that the pattern of disease has changed, with illnesses previously rare now ocurring in large cities. Most patients attending our clinic, including those with SLE, were from Harare. A formal epidemiological study is urgently needed to consider this problem.

Tropical polyarthritis has been one of the major causes of acute arthritis in Africa. ${ }^{411} 20$ This is a poorly defined entity and an identical clinical picture may be caused by gonococcal arthritis or poststreptococcal reactive arthritis. ${ }^{21}$ We saw only four patients with an acute arthritis that did not fulfil the criteria for well recognised diagnostic categories. These patients might have had tropical polyarthritis or a postviral arthritis. Possible reasons for the rarity of tropical polyarthritis at our clinic are that patients are often incapacitated and admitted to 
hospital, or again the pattern of disease may be changing. A formal study of patients admitted to hospital with arthritis is under way to answer this question. Osteoarthritis, particularly involving the knees, is not uncommon. A referral pattern biased towards the inflammatory arthritides is the explanation for the few patients seen.

The spondyloarthropathies are rare in Africa with less than 20 cases of ankylosing spondylitis reported before $1980,{ }^{22}$ and Reiter's syndrome recorded in isolated case reports. ${ }^{23} 24$ Striking features in our patients were the absence of both HLA-B27 and a family history of arthritis. ${ }^{18}$

We have described the pattern of rheumatic diseases seen at a specialist clinic in Harare. This differs from most other reports in that tropical polyarthritis is uncommon whereas the spondyloarthropathies and connective tissue diseases are being seen more often.

1 Anonymous. What can be learned from third world rheuma- I tism? [Editorial]. $\mathrm{Br} \mathcal{F}$ Rheumatol 1987; 26: 1-4.

2 Hall L. Polyarthritis in Kenya. East Afr Med $\mathscr{J}$ 1966; 43: 161-70.

3 Percy-Lancaster R. Arthritis survey in Transkei and Ciskei. $S$ Afr Med F 1974; 48: 2355-6.

4 Greenwood B M. Polyarthritis in Western Nigeria. I. Rheumatoid arthritis. Ann Rheum Dis 1969; 28: 488-96.

5 Solomon L, Beighton P, Valkenburg H A, Robin G, Soskolne $C$ L. Rheumatic disorders in the South African Negro. Part I. Rheumatoid arthritis and ankylosing spondylitis. S Afr Med J 1975; 49: 1292-6.

6 Kanyerezi B R, Lutalo S K. Some aspects of rheumatoid disease in Uganda. East Afr Med $\mathcal{F}$ 1980; 57: 39-43.
7 Bagg L R, Hansen D P, Lewis C, Houba V. Rheumatoid Bagg L R, Hansen D P, Lewis C, Houba V. Rheumatoid
arthritis in Kenya. I Clinical observations. Ann Rheum Dis arthritis in Keny.

8 Moolenburgh J D, Moore S, Valkenburg H A, Erasmus M G. Rheumatoid arthritis in Lesotho. Ann Rheum Dis 1984; 43: $40-3$.

9 Trowell H C. Non-infective disease in Africa. London: Arnold, 1960: 145 .

10 Taylor H G, Stein C M. Systemic lupus erythematosus in Zimbabwe. Ann Rheum Dis 1986; 45: 645-8.

11 Gelfand M. Medical arthritis in African practice. Cent Afr $\mathcal{F}$ Med 1969; 15: 131-5.

12 Lutalo $S \mathrm{~K}$. Chronic inflammatory rheumatic diseases in black Zimbabweans. Ann Rheum Dis 1985; 44: 121-5.

13 Ropes M W, Bennett G A, Cobb S, Jacox R, Jessar R A. Revision of diagnostic criteria in rheumatoid arthritis. Bull Rheum Dis 1959; 9: 175-6.

Rheum Dis 1959; 9: 175-6.
14 Tan E M, Cohen A S, Fries J F, et al. The 1982 revised criteria for the classification of systemic lupus erythematosus. Arthritis Rheum 1982; 25: 1271-7.

15 Calin A. Reiter's syndrome. In: Kelly W N, Morris E D, Ruddy S, Sledge C B, eds. Textbook of rheumatology. Philadelphia: Saunders, 1985: 1007-20.

16 Bennett P H, Wood P N H. Third international symposium on population studies of the rheumatic diseases. Amsterdam: Excerpta Medica, 1968: 456.

17 Steinbrocker O, Traeger C H, Batterman R C. Therapeutic criteria in rheumatoid arthritis. $\mathcal{F A M A} 1949 ; 140: 659-62$.

18 Stein M, Davis P, Emmanuel J. Immunogenetic profile of patients with ankylosing spondylitis and Reiter's syndrome patients with ankylosing spondylitis and Reiter's syndrome (suppl 2): 78 .

19 Davis P, Stein M, Latif A S, Emmanuel J. HIV and polyarthritis. Lancet 1988; i: 936.

20 Riley M J. Acute non-specific polyarthritis. Cent A/r f Med 1976; $22: 1-4$.

21 Stein $C M$, Hanly $M$ G. Acute tropical polyarthritis in Zimbabwe: a prospective search for a gonococcal aetiology. Ann Rheum Dis 1987; 46: 912-4.

22 Chalmers I. Ankylosing spondylitis in African blacks. Arthritis Rheum 1980; 23: 1366-70.

23 Methven P G, Swift P J. Reiter's syndrome in a black patient: a case report. $S$ Afr Med $\mathcal{F}$ 1985; 68: 597-8.

24 Seedat $\mathrm{Y}$ K Duminiet $\mathrm{H}$. Reiter's syndrome in a black patient: a case report. $S$ Afr Med F 1982; 61: 289. 